\title{
Motivos de inaptidão de candidatos à doação de sangue em hemocentro brasileiro
}

\section{Reasons for candidates inability for blood donation in a Brazilian blood center}

Elaine Drehmer de Almeida Cruz ${ }^{1}$, Magali Zimmermann Covo², Aline Batista Maurício ${ }^{3}$

1. ORCID: https://orcid.org/0000-0002-7686-6340. Doutorado. Universidade Federal do Paraná (UFPR), Programa de Pós-Graduação em Enfermagem. Curitiba, Paraná, Brasil. E-mail: elainedrehmercruz@gmail.com.

2. ORCID: https://orcid.org/0000-0001-5298-971X. Mestrado. Centro de Hematologia e Hemoterapia do Paraná (HEMEPAR). Curitiba, Paraná, Brasil. E-mail: magazmm@gmail. com

3. ORCID: https://orcid.org/0000-0002-6506-3737. Mestrado. Universidade Federal do Paraná (UFPR), Programa de Pós-Graduação em Enfermagem. Curitiba, Paraná, Brasil. E-mail: alinebatista.abm@gmail.com.

CONTATO: Autor correspondente: Magali Zimmermann Covo | Endereço: Rua José Luiz Demeterco, 1173 Ap.08. Telefone: (41) 999406150. E-mail: magazmm @gmail.com.

RESUMO Inaptidões clínicas em serviços de coleta de sangue correspondem a recusas de candidatos. Este estudo teve como objetivo identificar as causas e a frequência de inaptidões de candidatos à doação de sangue no Hemocentro Coordenador do Paraná. Fez-se análise exploratória e documental com dados do período de 2010 a 2015, coletados do Sistema Hemovida e relatórios do hemocentro; os dados foram analisados por estatística descritiva. Dos 220.496 candidatos que se apresentaram no período estudado, 30.950 $(14,0 \%)$ foram classificados como inaptos à doação de sangue. $E$ as causas mais frequentes encontradas entre eles foram risco para transmissão de doenças $(36,6 \%)$, sinais e sintomas clínicos $(23,4 \%)$ e exclusão médica $(9,9 \%)$. 
Considerando o ônus aos candidatos e ao hemocentro, ações coordenadas são necessárias para prevenir e reduzir a prevalência da inaptidão.

DESCRITORES: Doadores de Sangue. Bancos de Sangue. Seleção do Doador. Serviço de Hemoterapia.

ABSTRACT Clinical inadequacies in blood collection services correspond to candidate refusals. This study aimed to identify the causes and frequency of candidates inability for blood donation at the Coordinating Blood Center of $\mathrm{Pa}$ raná. An exploratory and documental analysis was carried out with data from 2010 to 2015, collected from the Hemovida System and reports from the blood center; data were analyzed using descriptive statistics. Of the 220,496 candidates who presented themselves during the study period, 30,950 (14.0\%) were classified as unsuitable for blood donation. And the most frequent causes found among them were risk for disease transmission (36.6\%), clinical signs and symptoms (23.4\%) and medical exclusion (9.9\%). Considering the burden on candidates and the blood center, coordinated actions are needed to prevent and reduce the inability prevalence.

DESCRIPTORS: Blood Donors. Blood Banks. Donor Selection. Hemotheraphy Service. 


\section{INTRODUÇÃO}

A Organização Mundial de Saúde (OMS) indica que das 118,5 milhões de doações de sangue no mundo, $40 \%$ ocorrem em países de alta renda, onde residem $16 \%$ da população mundial ${ }^{1}$. Ao considerar uma amostra de mil pessoas, a taxa de doação de sangue é de $31,5 \%$, em países de alta renda, de 15,9\%, naqueles de renda média alta, de $6,8 \%$, em países de renda média baixa e $5,0 \%$, em países de baixa renda 1 . O Ministério da Saúde brasileiro estima que somente 16 a cada mil habitantes são doadores de sangue, o que corresponde a $1,6 \%$ da população; e destas, $66 \%$ são classificadas como doações voluntárias ${ }^{2}$.

O estado do Paraná registrou, em 2016, o índice de $2 \%$ de doações entre a população, o qual se encontra aquém do estabelecido pelo Plano Diretor do Sangue, Hemocomponentes e Hemoderivados estadual, cuja meta para o período era de $2,5 \%$, com vistas a atender os usuários do Sistema Único de Saúde ${ }^{3}$.

No Brasil, a Hemoterapia segue legislação estabelecida pela Agência Nacional de Vigilância Sanitária (ANVISA) e, neste sentido, o Hemocentro Coordenador do Paraná (Hemepar) atende às normas da Portaria de Consolidação $n^{\circ} 5$, de 28 de setembro de $2017^{4}$, que abrange todo o ciclo do sangue, desde a observação de diretrizes para o cadastro de doadores às etapas subsequentes, como triagens clínica e hematológica, coleta, processamento e transfusão sanguínea.

A triagem clínica do candidato à doação é realizada em ambiente individual e por profissional habilitado, que decide pela aptidão ou inaptidão frente às condições de risco para o potencial doador e para o receptor. $\mathrm{Na}$ etapa seguinte, de triagem laboratorial, caso identificada inaptidão sorológica, o doador é contatado para esclarecimentos e encaminhamento do caso, e o sangue coletado é desprezado. Apesar de existirem exames que identifiquem algumas doenças, para muitas outras não é possível atestar a positividade sorológica, como se observa para a Doença de Creutzfeldt-Jakob, cuja inaptidão é definitiva ${ }^{4}$. Outrossim, no estado do Piauí observou-se casos de Doença de Chagas, no período de 2004 a 2013, com frequência de 58,6\% em 220 municípios. Houve encaminhamento de $34,5 \%$ das amostras positivas para testes complementares e, entre estas, $84,4 \%$ não se confirmaram, apontando a possibilidade da transmissão vetorial ${ }^{5}$. Outra pesquisa naquele estado avaliou a prevalência das principais doenças investigadas na triagem sorológica de quatro hemocentros em 2012. Das 49.829 doações sanguíneas, 1.818 foram bloqueadas na triagem sorológica de doadores com idade entre 17 e 67 anos, sobretudo para Hepatite B. Observou-se valores elevados quando comparados aos parâmetros nacionais, destacando-se a importância de campanhas educativas no contexto da prevenção de doenças, identificação de casos e conhecimento da inaptidão permanente à doação ${ }^{6}$. 
Os elevados índices de inaptidão clínica e sorológica podem ser determinantes para baixos estoques de sangue nos hemocentros. Considera-se que estas causem inúmeros constrangimentos e transtornos e, de forma geral, a falta de informação prévia é determinante. No Paraná, o candidato a ser atendido tem os dados consultados no Sistema Estadual de Informação e Controle Hemoterápico (SHTweb), que possibilita o acompanhamento/controle das doações e transfusões no estado, e representa aspecto positivo ${ }^{3}$. Esse sistema possibilita o acompanhamento/controle das doações e transfusões, com o objetivo de manter a vigilância da qualidade de sangue e hemocomponentes. As causas de inaptidão são classificadas no sistema e constituem ferramentas para a identificação das principais ocorrências, com o intuito de intensificar ações educativas aos candidatos à doação de sangue, assim como auxiliar nas políticas públicas.

O Hemepar é responsável pela coordenação da Política Estadual de Sangue nos serviços públicos de Hemoterapia que prestam atendimento ao SUS no Paraná; realiza o planejamento, a programação e a supervisão das atividades de captação de doadores, coleta de sangue, triagem laboratorial, produção e distribuição de hemocomponentes ${ }^{7}$. A hemorrede estadual presta assistência ambulatorial a, aproximadamente, 2.265 usuários portadores de coagulopatias e hemoglobinopatias hereditárias. O Hemepar é referência nacional em Imunohematologia e disponibiliza o Programa de Doadores Fenotipados, com mais de 40 mil doadores cadastrados ${ }^{7}$.

Neste contexto, esta pesquisa teve como objetivo identificar as causas de inaptidão de candidatos à doação de sangue no Hemepar, bem como a sua frequência.

\section{MÉTODO}

Tratou-se de pesquisa exploratória, documental de abordagem quantitativa. Foram delimitados para o estudo os dados relativos ao período de 2010 a 2015, em planilhas do Hemepar, construídas a partir do Sistema Hemovida, e no Relatório de Atividades Institucional, que contém os dados do Sistema Nacional de Informação da Produção Hemoterápica - Hemoprod, alimentado mensalmente. O Sistema Hemovida foi desenvolvido e implantado em 1999, especificamente para bancos de sangue, e teve por objetivo informatizar os dados do ciclo do sangue do Brasil, controlando cada etapa do processo e permitindo aos gestores acessar plenamente os dados indispensáveis à tomada de decisões ${ }^{8}$. Porém, esse sistema foi descontinuado a partir de 2016, ocasionando mudanças no conteúdo das planilhas, justificando-se o recorte temporal nesta pesquisa.

Os critérios de inclusão do doador no estudo foram: ter comparecido ao hemocentro no período de 2010 a 2015, ter realizado o cadastro para a doação e ter realizado consulta de triagem clínica; não foram previstos critérios de exclusão. Demais variáveis de interesse ao estudo foram extraídas de relatórios do Hemepar e incluídas em planilha do programa Microsoft Office Exce/®, por dupla digitação, seguida de conferência e correção 
de inconsistências. Após a síntese das informações, os dados foram complementados com informações do Relatório de Atividades Institucional relativas ao período estudado, analisados por estatística descritiva e apresentados em frequências absoluta e relativa.

O estudo seguiu os preceitos éticos e foi aprovado pelo Comitê de Ética em Pesquisa com Seres Humanos do Hospital do Trabalhador - Secretaria de Estado da Saúde do Paraná, sob protocolo número 1.898.252/2017.

\section{RESULTADOS}

Do total de 220.496 candidatos à doação de sangue no Hemepar no período de 2010 a $2015,30.950(14 \%)$ foram considerados inaptos e $189.546(86 \%)$ aptos. Os dados estão apresentados na Tabela 1, de acordo com o tipo de doação. Observa-se maior frequência de voluntários $(79,4 \%)$, sendo aptos, $85,9 \%$ desses. Porém, a aptidão teve maior frequência entre os convocados (93\%), que correspondem àqueles fenotipados ou "padrinhos do sangue" de pacientes do Ambulatório de Atendimento às Coagulopatias e Hemoglobinopatias.

Entre candidatos de reposição, o número de inaptidões foi maior $(15,2 \%)$, justificando-se que esse tipo de doação é motivado pela necessidade de restituição do sangue utilizado. Já o índice de candidatos à autotransfusão mostra-se insignificante quando comparado aos demais; porém, registrou $20 \%$ de inaptidão, indicando impedimentos de alguns candidatos à doação, os quais são também receptores.

Tabela 1. Perfil dos candidatos à doação no Hemocentro Coordenador do Estado do Paraná. Curitiba, PR, Brasil, 2010-2015.

\begin{tabular}{lccc}
\hline VARIÁVEIS & $\begin{array}{c}\text { Aptos } \\
\mathbf{N ~ ( \% )}\end{array}$ & $\begin{array}{c}\text { Inaptos } \\
\mathbf{N ~ ( \% )}\end{array}$ & $\begin{array}{c}\text { Total } \\
\mathbf{N}(\%)\end{array}$ \\
\hline \multirow{2}{*}{ Voluntários } & 150.395 & 24.765 & 175.160 \\
& $(85,9)$ & $(14,1)$ & $(79,4)$ \\
Reposição & 31.270 & 5.586 & 36.856 \\
& $(84,8)$ & $(15,2)$ & $(16,7)$ \\
Convocados & 7.861 & 594 & 8.455 \\
& $(93,0)$ & $(7,0)$ & $(3,8)$ \\
Autotransfusão & 20 & 5 & 25 \\
Total & $(80,0)$ & $(20,0)$ & $(0,0)$ \\
& $\mathbf{1 8 9 . 5 4 6}$ & $\mathbf{3 0 . 9 5 0}$ & $\mathbf{2 2 0 . 4 9 6}$ \\
\hline
\end{tabular}

Fonte: Adaptado do HEMOVIDA ${ }^{8}$ 
A Tabela 2 apresenta as causas registradas de inaptidão à doação de sangue, correspondendo a 13,9\% em relação ao número total de candidatos. Apresenta-se a classificação de 30.569 inaptidões, valor diferente do encontrado na Tabela 1 (30.950 inaptos), evidenciando que em 381 casos o motivo da inaptidão não foi informado no sistema Hemovida ${ }^{8}$. A classificação das causas de inaptidão segue o preconizado pela legislação vigente no período do registro dos dados no sistema.

O motivo mais frequente de inaptidão foi "risco para transmissão de doenças" (36,6\%), seguido por "sinais e sintomas clínicos" $(23,4 \%)$, que incluem variações de pressão arterial, de hemoglobina e alergias.

Tabela 2. Frequência das causas de inaptidão registradas no Hemocentro Coordenador do Estado do Paraná. Curitiba, PR, Brasil, 2010-2015.

\begin{tabular}{lcc}
\hline Causas de Inaptidão & $\mathbf{N}$ & \% \\
\hline Risco para transmissão de doenças & 11.194 & 36,6 \\
Sinais e sintomas clínicos & 7.154 & 23,4 \\
Exclusão médica & 3.018 & 9,9 \\
Outras patologias de inaptidão temporária & 2.802 & 9,2 \\
Procedimentos com pequenos sangramentos & 2.106 & 6,9 \\
Uso de medicação & 2.004 & 6,6 \\
Peso inferior a 50 quilogramas & 580 & 1,9 \\
Doenças que restringem a doação & 560 & 1,8 \\
Imunização ou uso de soro & 366 & 1,2 \\
Outras patologias de inaptidão definitivas & 239 & 0,8 \\
Motivos ginecológicos ou obstétricos & 180 & 0,6 \\
Risco pós doação/motorista & 119 & 0,4 \\
Transfusão de sangue há menos 10 anos & 101 & 0,3 \\
Número completo de doações em 12 meses & 57 & 0,2 \\
Uso de álcool & 30 & 0,1 \\
Candidatos aprazados & 29 & 0,1 \\
Consta na relação de doadores inaptos definitivos & 14 & 0,0 \\
Candidatos fora da faixa etária permitida & 8 & 0,0 \\
Sem rejeição autotransfusão/ aférese & 4 & 0,0 \\
Inacessibilidade de veias & 3 & 0,0 \\
Aguardando sorologia & 1 & 0,0 \\
Total & 30.569 & 100,0 \\
\hline Fon:Adapta & &
\end{tabular}

Fonte: Adaptado de HEMOVIDA ${ }^{8}$

\section{DISCUSSÃO}

Candidatos de reposição se apresentam ao hemocentro motivados a auxiliar familiares ou amigos que receberam sangue, nem sempre cientes das principais causas de inaptidão e da adequada orientação oferecida pelo serviço solicitante. Nesta modalida- 
de, orienta-se o cuidado na captação de doadores que, embora sensibilizados a doar, se apresentam em condições que podem comprometer a doação segura, como portadores de Diabetes tipo I, de doença cardíaca ou infecciosa. A OMS enfatiza que o suprimento adequado e confiável de sangue pode ser garantido por base estável de doadores regulares, voluntários e não remunerados, já que a prevalência de infecções por sangue é menor nesse grupo ${ }^{1}$.

Observou-se elevado índice de inaptidões classificadas nesta pesquisa $(13,9 \%$, $\mathrm{N}=30.569$ ), e a lacuna de 381 inaptidões sem classificação representou fragilidade no sistema. Observa-se dados subutilizados para seus fins, tendo como causa a baixa qualidade do preenchimento de informações, excesso de formulários, falta de conhecimento da importância dos sistemas empregados, e precariedade dos serviços de saúde em estrutura (tecnologia de informação) e pessoal, culminando em procrastinação no preenchimento de dados ${ }^{9}$.

O índice de candidatos inaptos (14\%) mostrou-se abaixo dos $20 \%$ estabelecidos pela política pública nacional, conforme a última normativa publicada com meta para esse quesito ${ }^{10}$. Este resultado também se encontra abaixo da última meta, de $15 \%$, registrada pelo Plano Diretor do Sangue, Hemocomponentes e Hemoderivados ${ }^{3}$ com atualização para a hemorrede paranaense a cada quatro anos.

A causa mais frequente de inaptidão (risco para transmissão de doenças) inclui condições nas quais existe a possibilidade de doenças infectocontagiosas ou comportamentos que comprometem a segurança do sangue. Nesse aspecto, cabe ressaltar que a transmissão de doenças pela transfusão de sangue é um evento indesejado, e as ações para a sua prevenção devem ser iniciadas na triagem clínica. Com a implantação dos testes de ácidos nucleicos, a testagem de sangue se revelou benéfica em países onde a soroprevalência da transmissão de doenças, por meio de transfusão sanguínea, é alta, como é o caso da maioria dos países em desenvolvimento ${ }^{11}$. A implementação de testes sorológicos aumenta a segurança transfusional, porém, é limitada pela falta de vacinas específicas para vírus, como o da Hepatite $C$, doença concentrada entre usuários de drogas injetáveis, potenciais fontes de transmissão para a população em geral ${ }^{12}$.

Apesar da preocupação constante com o HIV, um estudo norte-fluminense analisou a prevalência das Hepatites B e C e constatou que 3,53\% dos doadores estavam infectados entre os anos de 2004 a 2015. Porém, ao longo do período observou-se declínio de 4,19\% em 2004 para 2,24\% em 2015, o que sugere a eficácia das medidas tomadas naquele contexto, e a melhora na informação aos doadores. As Hepatites B e C merecem atenção especial, pois nem sempre o diagnóstico é conhecido pelo portador ${ }^{13}$. 
A causa "sinais e sintomas clínicos" contempla diversos eventos onde há necessidade de adiar a doação, como hipertermia e manifestações alérgicas e gripais, sabidamente relacionadas à presença de microrganismos causadores de sintomas agudos, bem como alterações nos níveis pressóricos e de frequência cardíaca.

No Hemepar, os candidatos convocados (fenotipados) são cadastrados por solicitação própria, com a coleta de uma amostra de sangue e testagem de antígenos eritrocitários para a pesquisa de compatibilidade com o perfil sanguíneo de pacientes que recebem transfusão ambulatorial. Esses devem dispor de um grupo de fenotipados satisfatório, em função da grande variedade de sistemas e fenótipos identificados no sangue. Os testes imunohematológicos representam outra medida para a prevenção de incompatibilidades em pacientes politransfundidos, na medida em que as frequências fenotípicas são determinadas, e podem ser utilizadas pelos serviços de Hematologia e Hemoterapia na busca de unidades de concentrados de hemácias com fenótipos desejados, calculando-se a incidência de doadores compatíveis, em casos de receptores com múltiplos aloanticorpos ${ }^{14}$.

Outra forma de otimizar o uso do sangue é realizar a coleta conforme a necessidade, considerando os estoques disponíveis por tipo sanguíneo. Estudo realizado no Mato Grosso determinou a prevalência da distribuição de sistema ABO entre mulheres doadoras de sangue em uma unidade de coleta e transfusão, entre janeiro de 2014 e dezembro de 2015. O número de doadoras do grupo sanguíneo A foi crescente e do grupo $\mathrm{O}$, Rh positivo foi o mais frequente, encontrando semelhanças com outros estudos ${ }^{15}$.

O organizador de grupos de candidatos à doação, referência para as coletas internas e externas, nem sempre é a pessoa mais adequada para a orientação. Doadores, eventualmente, chegam em jejum e com peso corporal abaixo do preconizado, resultando em ônus para o candidato e para o sistema de saúde, sem atingir o objetivo. Estudo realizado em São Paulo concluiu que a produtividade da coleta externa representou a segunda área com menor produção; quando ajustado aos dias de funcionamento, apresentou a segunda maior produtividade entre todos os postos ${ }^{16}$. O pronto acesso à doação é fundamental para a redução de desistências ou inaptidões, na medida em que há stress pelo tempo de espera, descontrole de pressão arterial e glicemia.

O conhecimento das principais causas e frequência de inaptidão é essencial com vistas a potencializar o desempenho e reduzir custos evitáveis, padronizar protocolos e propiciar ambiente informativo no serviço de coleta de sangue. $O$ candidato inapto poderá, oportunamente, se tornar multiplicador de conhecimentos, o que permite a redução do desperdício com coletas inadequadas e promoção da satisfação dos usuários. Além das causas de inaptidão classificadas pelo Ministério da Saúde ${ }^{4}$, o hemocentro ainda enfrenta desafios para coletar sangue livre de doenças emergentes, reemergentes ou 
mesmo causadas por vetores. Assim, são necessárias medidas complementares como inaptidões pós viagens para locais onde a frequência de casos de determinada doença esteja aumentada.

A informação eficaz e prévia aos candidatos à doação de sangue é um desafio para o serviço de captação, visto que o uso de tecnologias precisa ser aprimorado, a exemplo da substituição das ferramentas, telefone e e-mail, pelo uso do chat e recebimento da carteirinha on-line. Também, a atualização na página virtual da instituição para consultas sobre os critérios para a doação de sangue, com destaque aos motivos de inaptidão, é fundamental.

\section{CONCLUSÃO}

Não houve inconsistências importantes nos dados coletados, porém, demonstrou-se possibilidade de melhora com perspectiva de adequações nos registros, que podem ser organizados e a classificação atualizada, sempre que necessário. Porém, observou-se 381 causas de inaptidão não registradas, o que representa potencial para melhorias.

A redução na frequência das inaptidões requer ações planejadas e estratégicas, bem como mudança gradual da cultura da própria população, enquanto cidadã e interessada em adquirir conhecimento sobre o processo de doação.

O conhecimento das principais causas e frequência de inaptidão é essencial com vistas a potencializar o desempenho do hemocentro e reduzir custos evitáveis, padronizar protocolos e propiciar ambiente informativo no serviço de coleta de sangue. $\mathrm{O}$ candidato inapto poderá, oportunamente, se tornar multiplicador de conhecimentos, o que permite a redução do desperdício com coletas inadequadas e promoção da satisfação dos usuários. Além das causas de inaptidão classificadas pelo Ministério da Saúde ${ }^{4}$, o hemocentro ainda enfrenta desafios para coletar sangue livre de doenças emergentes, reemergentes ou mesmo causadas por vetores. Assim, são necessárias medidas complementares como inaptidões pós viagens para locais onde a frequência de casos de determinada doença esteja aumentada.

Observa-se, como potencialidade, ações preventivas às inaptidões de candidatos, motivo constante de insatisfação e que gera custos à instituição. Os resultados deste estudo evidenciam grande variedade nas causas de inaptidão, propiciando diversidade de ações estratégicas, operacionais e educativas. 


\section{REFERÊNCIAS}

1. World Health Organization (WHO). Blood safety and availability [Internet]. Geneva: WHO; 2019. [acesso 2021 jan 18]. Disponível em: https://www.who.int/news-room/fact-sheets/detail/blood-safety-and-availability

2. Ventura C. Dezesseis a cada mil brasileiros doam sangue [Internet]. Brasília: Ministério da Saúde; 2019 [acesso em 2021 jan 17]. Disponível em: https://antigo.saude.gov.br/noticias/agencia-saude/45520-dezesseis-a-cada-mil-brasileiros-fazem-doacao-de-sangue

3. Secretaria de Estado da Saúde (SESA). Plano Diretor de sangue, componentes e hemoderivados, 2016 a 2019 [Internet]. Curitiba: SESA; 2016. [acesso 2021 jan 18]. Disponível em: https://www.saude.pr.gov.br/ Pagina/Hemepar-Centro-de-Hematologia-e-Hemoterapia-do-Parana

4. Brasil. Ministério da Saúde. Portaria de Consolidação $n^{\circ}$ 5, de 28 de setembro de 2017. Consolidação das normas sobre as ações e os serviços de saúde do Sistema Único de Saúde [Internet]. Brasília: Diário Oficial da União; 2017 [acesso em 2020 out 17]. Disponível em: https://portalarquivos2.saude.gov.br/images/pdf/2018/marco/29/PRC-5-Portaria-de-Consolida----o-n---5--de-28-de-setembro-de-2017.pdf

5. Santana MP, Souza-Santos R, Almeida AS. Prevalência da doença de Chagas entre doadores de sangue do Estado do Piauí, Brasil, no período de 2004 a 2013. Cad Saúde Pública [Internet]. 2018 [acesso em 2020 set 20]. Disponível em: http://dx.doi.org/10.1590/0102-311x00123716

6. Rodrigues AMX, Barbosa ML, Silva KMR, Santos JS, Costa ACR. Prevalência das principais doenças investigadas na triagem sorológica em unidades de um hemocentro [Internet]. Maranhão: Rev. Ciência \& Saberes. 2018 [acesso em 2020 jan 22]. Disponível em: http://www.facema.edu.br/ojs/index.php/ReOnFacema/article/view/377

7. Secretaria de Estado da Saúde (SESA). Conselho Estadual de Saúde. Plano Estadual de Saúde Paraná 2020 a 2023 [Internet]. Curitiba: SESA; 2020 [acesso em 2021 jan 16]. Disponível em: https://www.saude. pr.gov.br/Pagina/Plano-Estadual-de-Saude

8. Departamento de Informática do SUS (DATASUS). HEMOVIDA: Sistema de Gerenciamento em Serviços de Hemoterapia [Internet]. Brasília: Portal da Saúde; 2017 [acesso em 2020 jan 22]. Disponível em: http://datasus.saude.gov.br/sistemas-e-aplicativos/hospitalares/hemovida

9. Santos TO, Pereira LP, Silveira DT. Implantação de sistemas informatizados na saúde: uma revisão sistemática. Reciis - Rev Eletron Comun Inf Inov Saúde [Internet]. 2017 [acesso em 2020 jun 20]. Disponível em: https://doi.org/10.29397/reciis.v11i3.1064

10. Brasil. Ministério da Saúde. Portaria $n^{\circ} 1.101$, de 12 de junho de 2002 [Internet]. Brasília: Diário Oficial da União; 2002 [acesso em 2020 jul 20]. Disponível em: http://bvsms.saude.gov.br/bvs/saudelegis/ gm/2002/prt1101_12_06_2002.html

11. Kameda K, Corrêa MCDV, Cassier M. A incorporação do teste diagnóstico baseado na amplificação de ácidos nucleicos (Nat) para triagem de sangue no SUS: arranjos tecnológicos para a nacionalização do "NAT brasileiro". Physis [Internet]. 2018 [acesso em 2020 ago 05]. Disponível em: https://doi.org/10.1590/ s0103-73312018280108

12. Teles SA. Viral hepatitis: a challenge for nursing. Rev. Bras. Enferm. [Internet]. 2017 [acesso em 2020 set 16]. Disponível em: https://doi.org/10.1590/0034-7167.2017700201

13. Motté MO, Lopes ACDS, De Mello CL, Renon TF, Carneiro RDC, Freitas NA. Soroprevalência de infecção pelos vírus das hepatites $B$ e $C$ em doadores de sangue do norte-fluminense. Revista Científica da FMC [Internet]. 2018 [acesso em 2020 nov 13]. Disponível em: https://doi.org/10.29184/1980-7813. rcfmc.195.vol.13.n1.2018

14. Machado AC, Sell AM, Macedo LC, Reis PG, Visentainer JEL. Phenotypic frequencies of the Kell, Duffy and Kidd blood groups in blood donors from Apucarana Blood Center, southern Brazil. RBAC - Rev Brasil Anal Clín [Internet]. 2018 [acesso em 2020 nov 13]. Disponível em: http://fi-admin.bvsalud.org/document/ view/wup5z

15. Santos RF, Bordin RO, Alves SM, Medeiros MO. Prevalência da distribuição do sistema abo/rh entre mulheres doadoras de sangue na unidade de coleta e transfusão "Dr. Marcio Curvo de Lima" no município 
de Rondonópolis-MT. Biodiversidade [Internet]. 2019 [acesso 2020 jan 24]. Disponível em: https://periodicoscientificos.ufmt.br/ojs/index.php/biodiversidade/article/view/8245

16. Ohtani RF, Nascimento JOV, Nishimoto T, Mota NV y VP, Lambert S. Proposta de otimização da produtividade do setor de coleta externa de um hemocentro do Estado de São Paulo. Rev. Adm. Saúde [Internet]. 2018 [acesso em 2020 fev 24]. Disponível em: http://dx.doi.org/10.23973/ras.71.98

RECEBIDO: 22/02/2021

ACEITO: $12 / 07 / 2021$ 